\title{
ON CONVEX POWER SERIES OF A CONSERVATIVE MARKOV OPERATOR
}

\author{
S. R. FOGUEL AND B. WEISS
}

ABstract. A. Brunel proved that a conservative Markov operator, $P$, has a finite invariant measure if and only if every operator $Q=\sum_{n=0}^{\infty} \alpha_{n} P^{n}$ where $\alpha_{n} \geqq 0$ and $\sum \alpha_{n}=1$ is conservative.

In this note we give $a$ different proof and study related problems.

Introduction. We shall use the notation and definitions of [3]. Let us quote some basic results:

The operator $P$ is conservative if and only if for every $0 \leqq f \in L_{\infty}$ the sum $\sum_{n=0}^{\infty} P^{n} f$ assumes the values 0 or $\infty$ only.

The operator $P$ is conservative if and only if whenever $0 \leqq f \in L_{\infty}$ and $P f \leqq f$ then $P f=f$. See [4, Corollary 1].

If $P$ is conservative and $P f=f$, then $f$ is $\Sigma_{i}(P)$ measurable where $\Sigma_{i}(P)=$ $\left\{A: P 1_{A}=1_{A}\right\}$. See [3, Theorem A of Chapter III].

We shall study operators of the form $Q=\sum_{n=0}^{\infty} \alpha_{n} P^{n}$ where $\alpha_{n} \geqq 0$ and $\sum \alpha_{n}=1$. Such operators will be called convex power series of $P$, and denoted by $A(P)$ where $A(z)=\sum_{n=0}^{\infty} \alpha_{n} z^{n}$.

1. Conditions for $Q$ to be conservative.

THEOREM 1.1. Let $P$ be a conservative operator and $Q=A(P)$ a convex power series of $P$. If $\sum_{n=1} n \alpha_{n}<\infty$ then $Q$ is conservative too.

Proof. Note first that

$$
\sum_{n=0}^{\infty}\left(1-\alpha_{0}-\cdots-\alpha_{n}\right)=\sum_{n=0}^{\infty}\left(\sum_{k=n+1}^{\infty} \alpha_{k}\right)=\sum_{n=1}^{\infty} n \alpha_{n} .
$$

Put $\gamma_{n}=1-\left(\alpha_{0}+\cdots+\alpha_{n}\right)$ then, by assumption, $\sum \gamma_{n}<\infty$. Define $K=$ $\sum \gamma_{n} P^{n}$ then $K$ is a positive bounded operator on $L_{1}$. An easy computation shows that $I-Q=(I-P) K$. Thus if $0 \leqq f \in L_{\infty}$ and $(I-Q) f \geqq 0$ then $(I-P) K f \geqq 0$ and $(I-P) K f=0$ because $P$ is conservative. By the characterization of conservative operators, given in the introduction, $Q$ is conservative too.

REMARK. Every finite convex combination of $P^{k}$ is conservative.

Received by the editors December 17, 1971.

AMS (MOS) subject classifications (1970). Primary 28A65.

(C) American Mathematical Society 1973 
For the rest of this section we shall assume that $P$ is ergodic and conservative and study $\Sigma_{i}(Q)$. If $A \in \Sigma_{i}(Q)$ or $1_{A}=\sum \alpha_{n} P^{n} 1_{A}$ then whenever $\alpha_{n} \neq 0 P^{n} 1_{A} \leqq 1_{A}\left(\right.$ since $\left.P^{n} 1_{A} \leqq 1\right)$ and thus $P^{n} 1_{A}=1_{A}$ since $P^{n}$ is conservative.

Let $r$ be the maximal common divisor of $n$ such that $\alpha_{n} \neq 0$. Then, on the one hand, $Q=\sum_{n=0}^{\infty} \alpha_{n r} P^{n r}$, and on the other hand there exist $n_{1} \cdots n_{j}$ with $\alpha_{n_{k}} \neq 0$ and $n r=q_{1} n_{1}+\cdots+q_{i} n_{j}$ with $q_{i}$ positive integers and $n \geqq N$. Thus, $1_{A}=P^{(N+1) r} 1_{A}=P^{r} P^{N} 1_{A}=P^{r} 1_{A}$. To summarize $\Sigma_{i}(Q)=\Sigma_{i}\left(P^{r}\right)$ for some integer $r$.

LEMMA 1.2. Let $P$ be ergodic and conservative then $\Sigma_{i}\left(P^{r}\right)$ is atomic.

Proof. Let us assume, to the contrary, that $A_{n} \in \Sigma_{i}\left(P^{r}\right)$ where $A_{n} \downarrow \varnothing$. Now $0=\left(I-P^{r}\right) 1_{A_{n}}=(I-P)\left(I+P+\cdots+P^{r-1}\right) 1_{A_{n}}$. Since $P$ is ergodic and conservative $\left(I+P+\cdots+P^{r-1}\right) 1_{A_{n}}$ is a constant but $1_{A_{n}}(x)=1$ if $x \in A_{n}$. Thus $\left(I+P+\cdots+P^{r-1}\right) 1_{A_{n}} \geqq 1$ and this contradicts the continuity of $P$ on $L_{1}$.

Let us take an atom $A$, of $\Sigma_{i}\left(P^{r}\right)$. Put $P^{j} 1_{A}=f, 1 \leqq j \leqq r-1$. Note that $f \in \Sigma_{i}\left(P^{r}\right)$ too. Put $B_{\varepsilon}=\{x: f(x) \geqq \varepsilon\}, B_{0}=\{x: f(x)>0\}$; both sets are in $\Sigma_{i}\left(P^{r}\right)$. Now $f \geqq \varepsilon 1_{B_{\varepsilon}}$ thus $1_{A}=P^{r-j} f \geqq \varepsilon P^{r-j} 1_{B_{\varepsilon}}$ hence $P^{r-j} 1_{B_{\varepsilon}} \leqq 1_{A}$. Let $\varepsilon \rightarrow 0$ to conclude $P^{r-j} 1_{B} \leqq 1_{A}$. Since $A$ is an atom and $P^{r-j} 1_{B}$ is invariant under $P^{r}$, we must have $P^{r-j} 1_{B}=$ const $1_{A}$. Now $P^{r} 1_{B B}=1_{B}$ so the constant is one. Thus $P^{r-j} 1_{B}=P^{r-j} f$ but $1_{B} \geqq f$. Therefore, $\sum P^{n}\left(1_{B}-f\right)<\infty$ or $f=1_{B B}$.

ThEOREM 1.3. $\Sigma_{i}\left(P^{r}\right)=\left\{A_{0}, \cdots, A_{k-1}\right\}$ where the sets $A_{i}$ are disjoint, $k$ divides $r$ and $1_{A_{1}}=P 1_{A_{0}}, 1_{A_{2}}=P 1_{A_{1}}, \cdots, 1_{A_{0}}=P 1_{A_{k-1}}$.

Proof. Let $A_{0}$ be an atom of $\Sigma_{i}\left(P^{r}\right)$. By the above argument $P^{j} 1_{A_{0}}=1_{A}$ and $A_{j} \in \Sigma_{i}\left(P^{r}\right)$. Let $k$ be the first integer such that $P^{k} 1_{A_{0}}=1_{A_{0}}$. Clearly $k$ divides $r$ and the sets $A_{j}, 1 \leqq j \leqq k-1$, are disjoint: If $B \subset A_{j}$ and $B \in$ $\Sigma_{i}\left(P^{r}\right)$ then $P^{k-j} 1_{B} \leqq 1_{A_{0}}$ and like the previous argument must be equal to $1_{A_{0}}$ or $P^{k-j} 1_{B}=P^{k-j} 1_{A_{j}}$ and $B=A_{j}$.

Now $\bigcup_{j=0}^{k-1} A_{j}$ is invariant under $P$ and thus must be all of $X$. Clearly each $A_{i}$ is an atom of $\Sigma_{i}\left(P^{r}\right)$ and $\Sigma_{i}\left(P^{r}\right)=\left\{A_{0}, A_{1}, \cdots, A_{k-1}\right\}$.

REMARK. Note that if $n$ divides $m$ then $\Sigma_{i}\left(P^{n}\right) \subset \Sigma_{i}\left(P^{m}\right)$. Thus $\bigvee \Sigma_{i}\left(P^{n}\right)=\bigvee \Sigma_{i}\left(P^{n !}\right)$ and $\Sigma_{i}\left(P^{n !}\right)$ is monotone in $n$.

Theorem 1.3 was proved by Moy in [7, Theorem 1] for a more general case by a different method.

\section{Conditions for $Q$ to be dissipative.}

LEMMA 2.1. Let $P_{1}$ and $P_{2}$ be commuting elements of a Banach algebra with $\left\|P_{1}\right\|=\left\|P_{2}\right\|=1$. Let $Q=\alpha P_{1}+\beta P_{2}$ where $0<\alpha, \beta<1$ and $\alpha+\beta=1$. Then $\left\|Q^{n}\left(P_{1}-P_{2}\right)\right\| \leqq(K / \sqrt{ } n) \cdot \alpha \cdot \beta$ where $K$ is a constant. 
Proof. First let us reduce the problem to the case where $\alpha=\beta=\frac{1}{2}$ : If $\alpha<\frac{1}{2}$ then $Q=\frac{1}{2}\left(P_{1}^{\prime}+P_{2}\right)$ where $P_{1}^{\prime}=2 \alpha P_{1}+(1-2 \alpha) P_{2}$ and $P_{1}^{\prime}-P_{2}=$ $2 \alpha\left(P_{1}-P_{2}\right)$.

Following [8] we write

$$
\begin{aligned}
Q^{n}\left(P_{1}-P_{2}\right) & =\frac{1}{2^{n}} \sum_{k=0}^{n}\left(\begin{array}{l}
n \\
k
\end{array}\right) P_{1}^{k} P_{2}^{n-k}\left(P_{1}-P_{2}\right) \\
& =\frac{1}{2^{n}} \sum_{k=1}^{n}\left[\left(\begin{array}{c}
n \\
k-1
\end{array}\right)-\left(\begin{array}{l}
n \\
k
\end{array}\right)\right] P_{1}^{k} P_{2}^{n-k+1}+\frac{1}{2^{n}} P_{1}^{n+1}-\frac{1}{2^{n}} P_{2}^{n+-1}
\end{aligned}
$$

thus

$$
\left\|Q^{n}\left(P_{1}-P_{2}\right)\right\| \leqq \frac{1}{2^{n-1}}+\frac{1}{2^{n}} \sum_{k=1}^{n}\left|\left(\begin{array}{c}
n \\
k-1
\end{array}\right)-\left(\begin{array}{l}
n \\
k
\end{array}\right)\right|
$$

We may assume that $n$ is even. Since $\left(\begin{array}{l}n \\ k\end{array}\right)$ increases as $k$ increases from 0 to $n / 2$ and then decreases as $k$ goes from $n / 2$ to $n$ the sum of absolute values is bounded by $\left(2 / 2^{n}\right)\left(\begin{array}{c}n \\ n / 2\end{array}\right)$ which is, by Stirling's formula, bounded by $K / \sqrt{ } n$, and hence the lemma follows.

Let $P$ be an operator and $Q=\sum_{n=0}^{\infty} \alpha_{n} P^{n}, \alpha_{i} \geqq 0, \sum \alpha_{i}=1$. Assume that $\alpha_{i}$ and $\alpha_{j}(i<j)$ are the first nonzero coefficients. Put $Q^{\prime}=\sum \alpha_{n} P^{n-i}$ where $P^{i} Q^{\prime}=Q^{\prime} P^{i}=Q$. Choose $0<\gamma<\min \left\{\alpha_{i}, \alpha_{j}, \frac{1}{2}\right\}$. Then

$$
Q^{\prime}=\gamma\left(I+P^{j-i}\right)+\sum \beta_{n} P^{n}=\gamma\left(I+P^{j-i}\right)+Q_{1} \text {. }
$$

Note $\sum \beta_{n}+2 \gamma=1, \quad \beta_{n} \geqq 0$. Thus $Q^{\prime}=\frac{1}{2}\left[\left(2 \gamma+Q_{1}\right)+\left(2 \gamma P^{j-i}+Q_{1}\right)\right]$ and $\left\|Q^{\prime n}\left(I-P^{j-i}\right)\right\| \rightarrow 0$. Therefore, $\left\|Q^{n}\left(I-P^{j-i}\right)\right\|=\left\|P^{i n} Q^{\prime n}\left(I-P^{j-i}\right)\right\| \rightarrow 0$.

THEOREM 2.2. Let $P$ be an operator with no invariant measure. Let $Q=$ $A(P)$ be a convex power series of $P$ such that $A(z)$ has at least two nonzero coefficients. There exists a set $A, m(A)>0$, such that $\left\|Q^{n} 1_{A}\right\|_{\infty} \rightarrow 0$.

Proof. Note first that, by a standard argument, $P^{j-i}$ has no invariant measure. By [3, Chapter IV, (4.9)] there exists a set $A$ with $m(A)>0$, such that if $\lambda P^{j-i}=\lambda$ then $\lambda(A)=0\left(\lambda \in L_{\infty}^{*}\right)$. By the Hahn-Banach Theorem

Thus $\left\|Q^{n} 1_{A}\right\|_{\infty} \rightarrow 0$.

$$
1_{A} \in \overline{\operatorname{Range}\left(I-P^{j-i}\right)} \text {. }
$$

REMARK. In [1, Lemma 1] Brunel proves that property for $Q=$ (1/e)exp $P$.

THEOREM 2.3. Let $Q$ be a Markov operator such that, for some $0 \leqq h \in$ $L_{\infty},\left\|Q^{n} h\right\| \rightarrow 0$. Choose a sequence $N_{m}$ such that $\sum_{m=0}^{\infty}\left\|Q^{N_{m}} h\right\|<\infty$. The operator $R=\sum \alpha_{n} Q^{n}$ is not conservative if $\sum_{m=0}^{\infty}\left(\sum_{n=0}^{N_{m}^{-1}} \alpha_{n}\right)^{m}<\infty$.

Proof. Put

$$
R=R_{1, m}+R_{2, m}=\sum_{n=0}^{N_{m}-1} \alpha_{n} Q^{n}+\sum_{n=N_{m}}^{\infty} \alpha_{n} Q^{n}
$$


Then $R^{m}=R_{1, m}^{m}+S_{m} Q^{N_{m}}$ where $S_{m}$ is of the form $\sum \beta_{n} Q^{n}, \sum \beta_{n} \leqq 1$, $\beta_{n} \geqq 0$. Thus

$$
R^{m} h=R_{1, m}^{m} h+S_{m} Q^{N_{m}} h \leqq R_{1, m}^{m} h+\left\|Q^{N_{m}} h\right\| .
$$

Since $\sum_{m=0}^{\infty}\left\|Q^{N_{m}}\right\|<\infty$ it is enough to consider the first term:

$$
R_{1, m}^{m} h=\left(\sum_{n=0}^{N_{m}-1} \alpha_{n} Q^{n}\right)^{m} h \leqq\|h\|\left(\sum_{n=0}^{N_{m}-1} \alpha_{n}\right)^{m}
$$

and the sum over $m$ of the right-hand side converges by assumption.

The Brunel Example. Let $\left\|Q^{n} h\right\| \rightarrow 0$ and $\sum\left\|Q^{N_{m}} h\right\|<\infty$. Choose

$$
\rho_{n}=\left(1 / n^{2}\right)^{1 / n}, \quad n \geqq 3,
$$

then $\rho_{n} \uparrow 1$ and $\sum \rho_{n}^{n}<\infty$. Choose $\alpha_{n} \geqq 0$ such that $\sum_{n=0}^{N_{m}-1} \alpha_{n}<\rho_{m}$ and $\sum \alpha_{n}=1$ and, by Theorem 2.3, $\sum \alpha_{n} Q^{n}$ is not conservative.

3. Dissipating power series. Let us call a power series $A(z)=\sum_{1}^{\infty} \alpha_{n} z^{n}$ dissipating if (1) $\alpha_{n} \geqq 0$, (2) $A(1)=1$, and (3) there is some conservative operator $P$ with $A(P)$ dissipative. Theorem 1.1 says simply that if $A^{\prime}(1)$ is finite then $A$ is not dissipating. The main purpose of this section is to establish a converse: namely if $A^{\prime}(1)$ is infinite then $A$ is dissipating. We first make a slight detour to discuss renewal sequences. Recall that $\left\{u_{n}\right\}_{n=1}^{\infty}$, $0 \leqq u_{n} \leqq 1$, is said to be a renewal sequence if there is a sequence $\left\{f_{n}\right\}_{n=1}^{\infty}$, $f_{n} \geqq 0, \sum_{1}^{\infty} f_{n} \leqq 1$ such that

$$
u_{n}=f_{n}+f_{n-1} u_{1}+f_{n-2} u_{2}+\cdots+f_{1} u_{n-1} \quad(n=1,2, \cdots) .
$$

Equivalently, if $U(z)=1+\sum u_{n} z^{n}, F(z)=\sum f_{n} z_{n}$ then

$$
U(z)=F(z) U(z)+1 \text { or } U(z)=\frac{1}{1-F(z)} .
$$

If $P=\left(p_{i j}\right)_{i, j=1}^{\infty}$ is a Markovian transition matrix with all states forming a single ergodic class, the condition for recurrence or conservativeness is simply $\sum_{n=0}^{\infty} p_{11}^{(n)}=+\infty$ where $p_{i j}^{(n)}$ is the $i j$ entry in $P^{n}$. It is well known that $\left\{p_{11}^{(n)}\right\}_{n=1}^{\infty}$ forms a renewal sequence. Here the $f_{n}$ of (1) represent the probability that first return to 1 takes place at time $n$. We shall need the simple converse.

LEMMA 3.1. If $\left\{u_{n}\right\}_{n=1}^{\infty}$ is a renewal sequence then there is an ergodic Markov matrix with $u_{n}=p_{11}^{(n)}$.

Proof. Let $f_{n}$ be such that (1) holds and define $p_{1}=f_{1}, \cdots, p_{n}=$ $f_{n} /\left(1-f_{1}-f_{2}-\cdots-f_{n-1}\right)$. Set now

$$
\begin{aligned}
p_{i j} & =p_{i} & & \text { if } j=1, \\
& =1-p_{i} & & \text { if } j=i+1, \\
& =0 & & \text { otherwise. }
\end{aligned}
$$


The lemma now follows easily if one recalls the probabilistic interpretation of the $f_{j}$ 's, namely that they are the probability that the event occurs for the first time at time $n$. The structure of $p_{i j}$ is such that one easily checks

Prob $\{$ return to 1 for the first time at time $n \mid$ start at 1$\}$

$$
=\left(1-p_{1}\right)\left(1-p_{2}\right) \cdots\left(1-p_{n-1}\right) p_{n}=f_{n} .
$$

The existence of a plentiful supply of renewal sequences is assured by Th. Kaluza's theorem [5] to the effect that if $1 \geqq u_{n} \geqq 0$ and

$$
u_{n} / u_{n-1} \leqq u_{n+1} / u_{n}, \quad n=1,2, \cdots \quad\left(u_{0}=1\right),
$$

then $\left\{u_{n}\right\}$ is a renewal sequence. Indeed as D. G. Kendall [6] has shown, these are precisely the "infinitely divisible" renewal sequences. We shall also need the following lemma, a proof of which may be found in [1].

LEMMA 3.2. If $x_{j}$ is a sequence of nonnegative numbers that tend to zero as $j \rightarrow \infty$ then there is a renewal sequence $\left\{u_{n}\right\}$, in fact, an infinitely divisible one, such that $\sum_{1}^{\infty} u_{n}=+\infty$ but $\sum_{1}^{\infty} u_{n} b_{n}<\infty$.

THEOREM 3.1. If $A(z)=\sum_{1}^{\infty} \alpha_{n} z^{n}, \alpha_{n} \geqq 0, A(1)=1$ and $A^{\prime}(1)=\infty$ then $A$ is dissipating.

Proof. Let $\beta_{j}$ be defined by

$$
\sum_{0}^{\infty} \beta_{j} z^{j}=\frac{1}{1-A(z)}=\sum_{0}^{\infty} A(z)^{n} .
$$

Then since $A^{\prime}(1)=1$ by the renewal theorem (see [2, Chapter XIII.3]) we know that $\beta_{j}$ tends to zero. Apply Lemma 3.2 to obtain a renewal sequence with $\sum_{1}^{\infty} u_{n}=\infty$ and $\sum_{1}^{\infty} u_{j} \beta_{j}<\infty$. By Lemma 3.1 there is a Markov matrix with $p_{11}^{(n)}=u_{n}$. Thus $P$ is conservative. However, $A(P)=Q$ is dissipative since

$$
\begin{aligned}
\sum_{0}^{\infty} Q_{11}^{(n)} & =\left(\sum_{0}^{\infty} Q^{n}\right)_{11}=\left(\sum_{0}^{\infty} A(P)^{n}\right)_{11}=\left(\sum_{0}^{\infty} \beta_{n} P^{n}\right)_{11} \\
& =\sum_{0}^{\infty} \beta_{n} p_{11}^{(n)}=\sum_{0}^{\infty} \beta_{n} u_{n}<+\propto .
\end{aligned}
$$

The formal interchanges of summations is justified since all the terms are nonnegative and the final result is a finite quantity.

It is worth remarking that even when a conservative operator $P$ has no finite invariant measure there are dissipating power series $A(z)$ such that $A(P)$ is conservative. To see this it suffices to consider the special translation invariant Markov chains on the integers $Z$-the random walks 
defined by $\left\{p_{j}\right\}$, a probability distribution on $Z$. A necessary and sufficient condition for recurrence is known here in terms of $\varphi(v)=\sum_{-\infty}^{\infty} p_{n} e^{i n v}$, the characteristic function of $\varphi$, namely

$$
\int_{-\pi}^{+\pi} \operatorname{Re}\left(\frac{1}{1-\varphi(v)}\right) d v=+\infty \quad[9, \text { Chapter II.8]. }
$$

Picking $p_{j}$ with prescribed behavior at infinity and using a Tauberian theorem to relate the behavior of $\varphi(v)$ at $\nu=0$ one readily produces examples for the phenomenon described above.

\section{BIBLIOGRAPHY}

1. A. Brunel, New conditions for existence of invariant measures in ergodic theory, Contributions to Ergodic Theory and Probability (Proc. Conf. Ohio State Univ., Columbus, Ohio, 1970), Springer-Verlag, Berlin and New York, 1970, pp. 7-17. MR 42 \#3253.

2. W. Feller, An introduction to probability theory and its applications. Vol. 1, 2nd ed., Wiley, New York, 1957. MR 19, 466.

3. S. R. Foguel, The ergodic theory of Markov processes, Van Nostrand, Princeton, N.J., 1969. MR 41 \#6299.

4. —_, Remarks on conservative Markov processes, Israel J. of Math. 6 (1968), 381-583. MR 39 \#4939.

5. Th. Kaluza, Über die Koeffizienten reziproker Potenzreihen, Math. Z. 28 (1928), 161-170.

6. D. G. Kendall, Renewal sequences and their arithmetic, Sympos. on Probability Methods in Analysis, Springer-Verlag, Berlin and New York, 1967, pp. 147-175. MR 36 \#7219.

7. S. T. C. Moy, Period of an irreducible operator, Illinois J. Math. 11 (1967), 24-39. MR 35 \#2350.

8. D. Ornstein and L. Sucheston, An operator theorem on $L_{1}$ convergence to zero with applications to Markov kernels, Ann. Math. Statist. 41 (1970), 1631-1639. MR 42 \#6938.

9. F. Spitzer, Principles of random walk, Van Nostrand, Princeton, N.J., 1964. MR 30 \#1521.

Department of Mathematics, Hebrew University, Jerusalem, Israel (Current address of $\mathbf{B}$. Weiss)

Current address (S. R. Foguel): Department of Mathematics, University of British Columbia, Vancouver 8, British Columbia, Canada 